\title{
Alimentación, crecimiento y supervivencia de Odontesthes bonariensis "pejerrey" hasta la etapa de alevines en condiciones de laboratorio
}

Feeding, growth and survival of the odonstheses bonairiensis "pejerrey"
up to the alevines stage in laboratory condition
Recibido: junio 07 de 2019 | Revisado: julio 15 de 2019 | Acepado: agosto 12 de 2019

Glicerio Reyes Amaru Chambilla ${ }^{\mathrm{I}}$

\section{Resumen}

En este trabajo determinamos la alimentación, crecimiento y supervivencia del Odontesthes bonariensis hasta la etapa de alevines en condiciones de laboratorio. Para determinar la viabilidad se suministró tres tipos de alimento vivo a larvas y alevinos de pejerrey, en donde la Chlorella vulgaris fue viable los primeros siete días. Posteriormente, la Artemia salina y Daphnia pulex resultaron ser viables en estado de neonatos. Mostraron diferencias estadísticamente significativas $\left(\mathrm{F}_{2.23}=\right.$ 0,0037; P- Value, 0.05), a un nivel de confianza al 95\%. En la frecuencia preferencial alimentaria observada en el cuarto día se encontró mayor presencia de Chlorella vulgaris al 60\%, menor viabilidad con Artemia salina al $40 \%$ y la Daphnia pulex no apareció como presa. Al analizar el tracto digestivo al día 11, la Chlorella representó al $10 \%$, Artemia salina al $80 \%$ y la Daphnia pulex al 10\%. La ocurrencia de dicho evento fue por el incremento de talla y peso del pez. Cabe resaltar que la Chlorella vulgaris fue relevante hasta los siete días. El índice de condición biológica en los tres ensayos fue proporcional a la talla y el peso hasta los 39 días con IK 1.43. A los 52 días, el índice incrementó significativamente IK 1.84 pudiendo estar dado por el cambio paulatino de alimento vivo a alimento balanceado inerte. Así mismo, la TCE en los primeros 13 días fue $9.9 \%$, posteriormente se mantuvo entre 2.71 a $4.15 \%$ (26 a 39 días), de 65 a 104 días la TCE se encontró entre 0.77 a $0.80 \%$ cercanos a 1 . Sin embargo, de 40 a 52 días la TCE fue la más bajo $0.60 \%$. La supervivencia en el E1 fue del $96.1 \%$, E2, 96\% y en el E3, 97.5 \%. Se concluyó que la alimentación, crecimiento y la supervivencia fueron satisfactorios en condiciones de laboratorio.

Palabras clave: Acuicultura, alimentación, crecimiento, pejerrey Odontesthes bonariensis

\section{Abstract}

The objective of the research was to determine the feeding, growth and survival of Odontesthes bonariensis up to the fry stage in laboratory conditions. To determine viability, three types of live food were fed to larvae and fingerlings of pejerrey, where Chlorella vulgaris was viable for the first seven days, and then Artemia salina and Daphnia pulex were found to be viable in neonatal status. They showed statistically significant

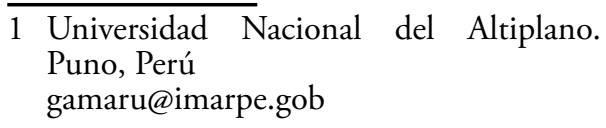

(C) Los autores. Este artículo es publicado por la Revista Campus de la Facultad de Ingeniería y Arquitectura de la Universidad de San Martín de Porres. Este artículo se distribuye en los términos de la Licencia Creative Commons Atribución No-comercial - Compartir-Igual 4.0 Internacional (https://creativecommons.org/licenses/ CC-BY), que permite el uso no comercial, distribución y reproducción en cualquier medio siempre que la obra original sea debidamente citada. Para uso comercial contactar a: revistacampus@usmp.pe. 
differences (F2.23 $=0.0037$, P-Value, 0.05), at a confidence level of $95 \%$. In the preferential food frequency observed on the fourth day; found a greater presence of Chlorella vulgaris at $60 \%$, lower viability with $40 \%$ Artemia salina and Daphnia pulex did not appear as prey. When analyzing the digestive tract on day 11 , Chlorella represented 10\%, Artemia salina $80 \%$ and Daphnia pulex $10 \%$, the occurrence of this event was due to the increase in size and weight of the fish. It should be noted that Chlorella vulgaris was relevant up to 7 days. The biological condition index in the three trials was proportional to the size and weight to 39 days with IK 1.43 , at 52 days, the index increased significantly IK 1.84, being able to be given by the gradual change of live food to inert balanced feed, likewise the TCE in the first 13 days was $9.9 \%$, later it stayed between 2.71 to $4.15 \%$ (26 to 39 days), from 65 to 104 days the TCE was between 0.77 to $0.80 \%$ close to 1. However, of 40 at 52 days the TCE was the lowest $0.60 \%$. The survival in E1 was 96.1\%, E2, 96\% and in E3, 97.5\%. It was concluded that feeding, growth and survival were satisfactory under laboratory conditions.

Key words: aquaculture, feeding, growth, pejerrey

Odontesthes bonariensis

\section{Introducción}

El pejerrey, Odontesthes bonariensis es uno de las especies de mayor importancia comercial en la región Puno y se ha constituido en un verdadero pilar de la economía de los habitantes de las riberas del lago Titicaca (Chura, et al 2013). El cultivo de esta especie se realizó en Argentina en la ciudad de Chascomús en el año 1904 (Tulian, 1909) y a partir de ese momento comenzó un programa de siembra en diversos cuerpos de agua provinciales, nacionales e internacionales debido a la gran importancia de su pesca comercial y deportiva (López \& García, 2001).

\section{La disminución de pejerrey Odontesthes} bonariensis en el lago Titicaca, según IMARPE 2017 llegó a - 11.6 \% entre los años 2016 a 2017. Dicha reducción sigue evidenciándose debido a una sobrepesca por una alta demanda del pejerrey en la Región Puno. De igual modo puede referirse que el uso de redes con luz de malla entre 0,64 y $1,27 \mathrm{~cm}$ ha afectado directamente el stock de los juveniles (Yujra et al., 2016).

Para una acuicultura sostenible de pejerrey es necesaria la producción masiva de larvas, manejo de alevinos y el uso adecuado de alimento; asimismo, es importante conocer la fisiología del aparato digestivo de esta especie en sus diferentes etapas para determinar el tipo de alimento a suministrar. Considerando que la alimentación y la nutrición de larvas de peces se inicia con la ingesta del alimento vivo, dichos alimentos contienen cantidades variables de proteínas, lípidos, vitaminas, minerales y otros compuestos incluyendo los que imparten aroma, sabor y color (Bdui, 1988).

A fin de asegurar la supervivencia de larvas de pejerrey es importante conocer el tipo de alimento a utilizar para cada etapa, dado que presentan una problemática especial al no tener un sistema digestivo completamente formado desde su eclosión; además deben atravesar por un 
proceso de transformación hasta la etapa juvenil, tiempo en que el pez completa el sistema de aparato digestivo, problema que se agudiza durante el periodo larvario ya que requieren alimentarse con presas vivas, menores a 06 micras (Tucker, 1998).

Las larvas de pejerrey presentan un régimen alimentario filtrador de plancton (Ringuelet, 1942; Burbidge et al., 1974; Bahamondes et al., 1979), razón por la cual los cultivos auxiliares desempeñan un papel importante en la acuicultura, principalmente, en el estadío larval del pejerrey y de algunos peces filtradores.

La fisiología digestiva de larvas de pejerrey aún no está bien estudiada, razón por la cual, se consideró necesario realizar un estudio sobre la alimentación en larvas $\mathrm{y}$ alevinos de pejerrey en condiciones controladas utilizando alimento vivo como: Chlorella vulgaris, Artemia salina y Daphnia pulex.

\section{Materiales y métodos}

La investigación fue realizada en las instalaciones del Laboratorio Continental de IMARPE-Puno, del barrio San Martín de la ciudad de Puno. El estudio se efectuó durante los meses de setiembre a diciembre del 2016. Para la investigación se utilizaron 6894 unidades de larvas de pejerrey de un día de edad, de las cuales, 900 unidades fueron utilizadas para el primer objetivo específico con tres repeticiones. Para el segundo y tercer objetivo fueron utilizados 5994 unidades de larvas de pejerrey confinadas en nueve acuarios de vidrio $\left(50 \times 40 \times 40 \mathrm{~cm}^{3}\right)$, por 15 días. Posteriormente, fueron trasladadas hacia tres tanques circulares de $0,5 \mathrm{~m}^{3}$, en ambos casos con sistema de aireación permanente y con un recambio de agua a cada cinco días.

Así mismo, para los tres ensayos se utilizaron métodos estadísticos, los cuales correspondieron al análisis de la varianza factorial y la prueba de intervalos múltiples de Tukey utilizando el software profesional Statgraphics (STATPOINT TECHNOLOGIES,

1994-2001) programa " $R$ " donde los resultados se consideraron significativos, a un nivel de confianza del 95\% ( $\mathrm{p} \leq 0.05)$.

\section{Determinación de la viabilidad alimentaria a base de Chlorella vulgaris, Artemia salina y Daphnia pulex a larvas $y$ alevinos de pejerrey}

Una vez obtenidas las larvas de pejerrey desde los vasos Chasse con volumen de agua de $5 \mathrm{~L}$ y con flujo continuo de 0.5 $\mathrm{L} / \mathrm{s}$ que funcionaron para cumplir con el proceso de incubación y eclosión, para este primer objetivo se utilizó 900 unidades de larvas con un peso promedio de $0.0010 \mathrm{~g}$ y con una longitud total de $0.70 \mathrm{~cm}$ del mismo cohorte los cuales fueron fraccionados en igual número de individuos en tres matraces de vidrio de un litro, tres muestreos por cada ensayo, el uso de matraz facilitó extraer y cuantificar con facilidad los peces muertos. Después de cuatro días se proporcionó a los peces alimento vivo dos veces al día, los cuales correspondieron a Chlorella vulgaris, Artemia salina y Daphnia pulex durante 11 días. Se determinó la viabilidad del tipo de alimento, porcentaje de mortalidad y supervivencia de larvas bajo el suministro de cada alimento vivo.

La proporción de Chlorella vulgaris a larvas de pejerrey fue $150 \mathrm{ml}$, con 
una densidad celular de 8 millones de unidades por $\mathrm{ml}$. El cultivo de esta especie se realizó en el laboratorio de cultivos auxiliares del laboratorio continental de IMARPE-Puno. La alimentación con Artemia salina fue dos veces al día, la cantidad suministrada fue de $3 \mathrm{ml}$, con una densidad de 100 unidades por $\mathrm{ml}$. Para la eclosión de sistos de Artemia salina, se pesó $5 \mathrm{~g}$ de quistes de Artemia salina el cual fue sometido en un envase con agua agitándolos por un periodo de 40 minutos.

Posteriormente, se le agregó hipoclorito de sodio al $6 \%(30 \mathrm{ml})$, el cual eliminó y desinfectó los agentes patógenos que pudieran estar presentes en los quistes de Artemia salina. Después de haberse hidratado y desinfectado los huevos de Artemia fueron enjuagados con agua potable y se procedió a incubarlos en un envase de un litro que contenía agua con una salinidad al $35 \%$, a una temperatura de $30{ }^{\circ} \mathrm{C}$ por un tiempo de 24 horas. En este lapso eclosionaron obteniendo nauplios de Artemia y estos fueron proporcionados a los peces dos veces al día. La cantidad de $2 \mathrm{ml}$ equivale a 20000 unidades. En cuanto a Daphnia pulex, se proporcionó $100 \mathrm{ml}$ a cada matraz con una densidad poblacional de ocho unidades por $\mathrm{ml} \mathrm{de}$ nauplios de Daphnia. Dichas Daphnias fueron capturadas de la bahía interior del lago Titicaca y fueron seleccionadas por tamańos mediante tamices y acondicionados en el laboratorio y alimentados con microalgas (Chlorella vulgaris y Scenedesmus sp).

La temperatura de los acuarios y tanques se mantuvo entre 16 a $17.4^{\circ} \mathrm{C}$, la concentración y el oxígeno disuelto entre 5.2 a $5.7 \mathrm{mg} / \mathrm{L}$.
Determinación de la frecuencia aparición de cada presa suministrados con Chlorella vulgaris, Artemia salina y Daphnia pulex, desde la etapa larvaria hasta alevines

De las 5994 unidades de larvas de pejerrey consideradas como población, se establecieron 1998 ejemplares para cada ensayo (tres réplicas). Estas fueron confinadas en nueve acuarios de vidrio $\left(50 \times 40 \times 40 \mathrm{~cm}^{3}\right)$, distribuidos a 666 larvas de pejerrey por acuario con aireación permanente por 15 días. Posteriormente, fueron trasladados a tres tanques circulares de $0,5 \mathrm{~m}^{3}$ previo control de parámetros físico-químicos, de igual forma con sistema de aireación y filtros para mantener limpia el agua. A partir del cuarto día de nacimiento se suministró a cada acuario tres variedades de alimento vivo; dos veces al día "ad libitum" Chlorella vulgaris, Artemia salina y Daphnia pulex. Para el análisis del contenido estomacal se utilizó 24 unidades de peces que correspondieron al tamaño de muestra, dicha actividad se efectuó cada siete días por un periodo de 46 días, el cual permitió corroborar con los resultados del primer objetivo.

Para determinar la frecuencia preferencial, ítems-presa se procedió a realizar la disección con un bisturí separando el tubo digestivo mediante un corte longitudinal. Posteriormente se extrajo el contenido del tracto digestivo con una pinza en una lámina porta objetos para su observación y análisis mediante el microscopio compuesto EUROMEX ISCOPE y estereoscopio KRUSS OPTRONIC. La identificación de los ítems-presa se realizó, a nivel de la especie y en función al grado de digestión de los mismos. 
Para determinar la frecuencia de aparición de cada presa, se calculó la frecuencia de ocurrencia (FO), a partir de la fórmula: $\mathrm{FO}=\mathrm{n} / \mathrm{N}^{*} 100$. Donde $\mathrm{n}=$ es el número de tractos digestivos que contiene una presa determinada, $\mathrm{N}$ = es el número total de tractos digestivos con alimento. A partir de los porcentajes obtenidos se distinguieron tres categorías de presas las que correspondieron a (Franco \& Bashirulah. 1992; Cardoza Martínez et al., 2011): (i) Alimento preferencial $(\mathrm{FO}>50)$ (ii) Alimento secundario $(10<\mathrm{FO}<50)$ y (iii) Alimento accidental $(\mathrm{FO}<10)$.

La limpieza de los acuarios se realizó cada dos días. Así mismo, el recambio de agua en los acuarios fue cada 10 días. Sin embargo, para peces confinados en tanques circulares el recambio de agua se efectuó cada cinco días, correspondiendo la tercera parte del total del volumen ya que experiencias propias indican que los recambios constantes, totales y cambios bruscos de temperatura ocasionan al pez estrés, atrofia muscular y muerte.

\section{Evaluación del crecimiento y supervivencia durante la etapa de larvas y alevinos bajo condición de laboratorio}

Se utilizaron muestras de peces a los cuales se midió la longitud total (LT) en centímetros, con Ictiómetro modelo PÉNTAIR y el peso en gramos con balanza de precisión Modelo PCE para relacionarlos y calcular el índice de condición biológica de Fulton (IK), a través de la siguiente fórmula: $\mathrm{IK}=\mathrm{P} / \mathrm{L}^{3}$ $\mathrm{x}$ 100. Dónde: $\mathrm{IK}=$ Índice de condición corporal de Fulton, $\mathrm{P}=$ Peso (gramos) y $\mathrm{L}=$ Longitud total $(\mathrm{cm})$. Dicho factor indica el estado nutritivo de una especie en cultivo en relación al medio en que vive en función a su nutrición. Así mismo, fue útil para comparar y cuantificar numéricamente la condición o estado del pez pudiendo asociarse a una valoración de la contextura o estado de delgadez o gordura (Martínez Millán, 1987). De similar forma, se estimó, la tasa de crecimiento específico (TCE) en porcentaje día. La relación longitudpeso se utilizó para estimar el peso correspondiente a una longitud dada mediante la siguiente fórmula (Ricker 1979): $\operatorname{TCE}(\% /$ día $)=(\ln \mathrm{XF}-\ln \mathrm{Xi})$ $/(\mathrm{T}) * 100$, donde $\ln \mathrm{XF}=$ logaritmo natural del peso húmedo final de la especie, $\ln \mathrm{Xi}=$ logaritmo natural del peso húmedo inicial de la especie $\mathrm{Y} T$ es el Tiempo (días). La ganancia del peso diario se determinó mediante la diferencia del peso final e inicial por el tiempo de cultivo. Al final del ensayo, se determinó la ganancia de peso total en gramos considerando el peso inicial el día cero y el final día (x) asumiendo que los peces aumentan de manera exponencial. En el crecimiento, supervivencia y mortalidad de alevinos de pejerrey, los factores influentes durante los ensayos fueron; la temperatura, oxígeno disuelto $\mathrm{pH}$, salinidad y amonio los cuales fueron controlados.

Para la evaluación de supervivencia de larvas y alevinos de Odontesthes bonariensis se realizó el conteo de individuos muertos y el porcentaje que representa a todos los acuarios y en tanques circulares. Las densidades que se manejaron fueron para acuarios de vidrios 15 ind/L, estabulados durante 30 días, posteriormente trasladadas a tanques circulares con una densidad y 5 ind/L, el agua utilizando para el cultivo fue de lago Titicaca, zona Ichu. 


\section{Resultados y discusión}

Determinación de la viabilidad alimentaria a base de Chlorella vulgaris, Artemia salina y Daphnia pulex a larvas y alevinos de pejerrey

Se consideró 300 individuos de larvas de pejerrey para determinar la viabilidad alimentaria. Se observó que al sétimo día, existió mayor viabilidad al suministrar Chlorella vulagris en los tres ensayos, obteniendo una supervivencia de 99, 95 y 90 individuos, mientras alimentados con Artemia salina la supervivencia fue de 74, 74 y 75 individuos y al suministrar Daphnia pulex la sobrevivencia ha sido de 45, 45 y 63 individuos. A partir del octavo día, la viabilidad con Chlorella vulgaris fue irrelevante puesto que los alevinos prefirieron alimentarse de Artemia salina y/o nauplios de Daphnia pulex probablemente por el tamaño de la presa debido a que el pez presentó incremento corporal en la talla y el peso con relación al tiempo (Tabla 1 ).

Se aprecia en las Tablas 2 al 7, correspondiente a los ensayos (1, 2 y 3 ), diferencias estadísticamente significativas $\mathrm{p} \leq 0.05$ entre los dietas proporcionadas y de acuerdo a la prueba de Tukey, se observó que la Chlorella vulgaris hubo diferencia respecto a Daphnia pulex $y$ Artemia salina a un nivel de confianza al $95 \%$.

Tabla 1

Viabilidad del tipo de alimento para la supervivencia larvaria de pejerrey, (ensayo 1,2 y3)

\begin{tabular}{cccccccccc}
\hline & \multicolumn{4}{c}{ Chlorella vulgaris } & \multicolumn{4}{c}{ Tipo de Alimento } \\
Día & E1 & E 2 & E3 & E1 & E 2 & E3 & E1 & E 2 & E3 \\
\hline 0 & 100 & 100 & 100 & 100 & 100 & 100 & 100 & 100 & 100 \\
4 & 100 & 99 & 97 & 90 & 94 & 90 & 80 & 79 & 82 \\
5 & 99 & 99 & 7 & 80 & 88 & 88 & 62 & 75 & 77 \\
6 & 99 & 99 & 95 & 78 & 74 & 85 & 48 & 75 & 75 \\
7 & 99 & 95 & 90 & 74 & 74 & 75 & 45 & 45 & 63 \\
8 & 95 & 95 & 88 & 73 & 73 & 75 & 43 & 43 & 60 \\
9 & 90 & 90 & 86 & 73 & 73 & 75 & 43 & 43 & 60 \\
10 & 88 & 87 & 84 & 71 & 73 & 75 & 43 & 43 & 59 \\
11 & 85 & 84 & 80 & 71 & 73 & 75 & 43 & 43 & 59 \\
\hline
\end{tabular}

$\mathrm{n}=900$, larvas de pejerrey para los tres tratamientos, $\mathrm{E}=$ ensayos

Tabla 2

Análisis de varianza. Preferencia alimentaria de larvas de pejerrey (Ensayo 1)

\begin{tabular}{cccccc}
\hline Fuente & Suma de Cuadrados & Gl & Cuadrado Medio & Cociente F & Valor-P \\
\hline Tratamientos & 7639.08 & 2 & 3819.54 & 44.70 & 0.0000 \\
Intra grupos & 1794.25 & 21 & 85.4405 & & \\
Total (Corr.) & 9433.33 & 23 & & & \\
\hline
\end{tabular}


AlimentaCión, CRECIMIENTO Y SUPERVIVENCIA DE ODONTESTHES BONARIENSIS “PEJERREY” HASTA LA ETAPA DE ALEVINES EN CONDICIONES DE LABORATORIO

Tabla 3

Prueba de Tukey. Preferencia alimentaria de larvas de pejerrey (Ensayo 1)

\begin{tabular}{lcc}
\hline Contraste & Sig & Diferencia \\
\hline Chlorella - Artemia salina & $*$ & 0.00041 \\
Chlorella - Daphnia pulex & $*$ & 0.00001 \\
Artemia salina - Daphnia pulex & $*$ & 0.00069
\end{tabular}

* indica diferencia significativa

Tabla 4

Análisis de varianza. Preferencia alimentaria de larvas de pejerrey (Ensayo2)

\begin{tabular}{lccccc}
\hline Fuente & Suma de Cuadrados & Gl & Cuadrado Medio & Cociente F & Valor-P \\
\hline Tratamientos & 5847.25 & 2 & 2923.63 & 21.42 & 0.0000 \\
Intra grupos & 2866.38 & 21 & 136.494 & & \\
\cline { 1 - 3 } Total (Corr.) & 8713.63 & 23 & & &
\end{tabular}

Tabla 5

Prueba de Tukey. Preferencia alimentaria de larvas de pejerrey (Ensayo 2)

\begin{tabular}{lcc}
\hline Contraste & Sig & Diferencia \\
\hline Chlorella - Artemia salina & $*$ & 0.0039 \\
Chlorella - Daphnia pulex & $*$ & 0.00027 \\
Artemia salina - Daphnia pulex & $*$ & 0.005 \\
\hline
\end{tabular}

* indica diferencia significativa

Tabla 6

Análisis de varianza. Preferencia alimentaria de larvas de pejerrey (Ensayo3)

\begin{tabular}{lccccc}
\hline Fuente & Suma de Cuadrados & Gl & Cuadrado Medio & Cociente F & Valor-P \\
\hline Tratamientos & 2094.33 & 2 & 1047.17 & 18.38 & 0.0000 \\
\hline Intra grupos & 1196.63 & 21 & 56.9821 & & \\
\cline { 1 - 4 } Total (Corr.) & 3290.96 & 23 & & &
\end{tabular}

Tabla 7

Prueba de Tukey. Preferencia alimentaria de larvas de pejerrey (Ensayo 3)

\begin{tabular}{lcc}
\hline Contraste & Sig & Diferencia \\
\hline Chlorella - Artemia salina & $*$ & 0.011 \\
Chlorella - Daphnia pulex & $*$ & 0.00001 \\
Artemia salina - Daphnia pulex & $*$ & 0.0058 \\
\hline
\end{tabular}

Las larvas de Odontesthes bonariensis eclosionan con saco vitelino y después de tres a cuatro días este se reabsorbe en su totalidad, de manera que, se admite 
como la primera tasa de alimentación. $\mathrm{Al}$ no presentar un sistema digestivo completamente formado hace que la alimentación sea dificultosa para determinados tipos de presas que posteriormente constituirán alimentos preferenciales. Es por ello que, los individuos después de tres días, requieren un tipo de alimento fácil de digerir, considerándose a esta primera etapa post-larvaria como planctófaga ya que es la única que puede ser incorporada sin afectar la vida de los alevinos de modo que, la Chlorella vulgaris, justificó el primer suministro en los tres ensayos, alcanzándose mejores indicadores sobre la viabilidad.

Govoni (1986) menciona que, en el momento de la eclosión, el sistema digestivo de las larvas es un simple tubo recto y corto sin mayor diferenciación. Durante el desarrollo y maduración del tracto digestivo, no se observan grandes cambios morfológicos hasta que inicia la formación del estómago con sus glándulas gástricas y los ciegos pilóricos. Por su parte Dabroswski (1984), indica que las larvas de pejerrey poseen una limitada cantidad de material vitelino, motivo del inicio temprano de alimentación exógena, aunque la capacidad digestiva es mínima, correspondiendo esta clasificación sobre la ecología y fisiología larvaria según el grado de desarrollo del sistema digestivo en el momento de reabsorción y alimentación exógena.

Ringuelet et al. (1980) señalaron que Odontesthes bonariensis se define como planctófago filtrador durante la primera etapa de vida, aunque posee placas faríngeas dentadas, indicando la posibilidad sobre un cambio de dieta en aquellos ambientes donde el plancton es escaso o inadecuado. y cuya precisión, pudo observarse en este trabajo de investigación donde los alevines mostraron una mejor viabilidad alimentaria con la Chlorella vulgaris en los tres ensayos, aunque de la misma manera, hubo actividad alimentaria sobre Artemia salina y la Daphnia pulex, lo cual corrobora lo planteado. Así mismo, consideró a la microalga como fuente directa de alimento, micronutrientes, suplemento de enzimas exógenas que ayudan en la digestión, y estímulo directo de las enzimas.

Del Ponti \& García (2015) exhiben la composición porcentual discriminada por tallas de la alimentación de pejerrey e indican que la variedad de ítems presa hallados fue de carácter eurífago. Así mismo, Grosman (1995) reportó tres patrones temporales de alimentación, primero como planctófago, el segundo como micrófago y el tercero oportunista - macrófago, versiones con las cuales coinciden con el trabajo de investigación.

\section{Determinación de la frecuencia aparición de cada presa suministrados con Chlorella vulgaris, Artemia salina y Daphnia pulex, desde la etapa larvaria hasta alevines}

Al observar el sistema digestivo del pez en el cuarto día, se encontró Cholrella vulgaris al 60\%, Artemia salina al 40\%. $\mathrm{Al}$ onceavo día, la aparición de alimentos en el sistema digestivo fue la siguiente: Chlorella vulgaris 10\%, Artemia salina $80 \%$ y Daphnia pulex $10 \%$, y al día 18, se encontró $70 \%$ de Artemia salina y 30\% de Daphnia pulex. No se encontró la microalga Chlorella vulagris. A partir del día 32, los peces necesitaron alimentarse de presas de mayor tamaño 
$\mathrm{y}$ es en este periodo donde se aprecia que la especie Daphnia pulex resultó ser alimento adecuado durante los últimos 14 días. Estos resultados mostraron que los alevinos de Odontesthes bonariensis, demandan diferencias en el tipo de alimento de acuerdo al tamaño del pez (Tabla 8 y Figura 1)

Tabla 8

Frecuencia de aparición alimentaria en el sistema digestivo de pejerrey

\begin{tabular}{|c|c|c|c|}
\hline Día & Chlorella v. & Artemia salina & Daphnia pulex \\
\hline 4 & $F o=60$ & $F o^{*}=40$ & \\
\hline 11 & $F o=10$ & $F 0^{*}=80$ & $F o^{*}=10$ \\
\hline 18 & & $F o^{*}=70$ & $F o^{* *}=30$ \\
\hline 25 & & $F o^{*}=50$ & $F o^{* *}=50$ \\
\hline 32 & & $F o^{* *}=40$ & $F o^{* *}=60$ \\
\hline 39 & & $F o^{* *}=30$ & $F_{0}^{* *}=70$ \\
\hline 46 & & $F^{* * *}=20$ & $F_{0}^{* * *}=80$ \\
\hline
\end{tabular}

Figura 1. Frecuencia de aparición de presas durante los 46 días

Al analizar los tractos digestivos mediante el microscopio y estereoscopio, se presenció Chlorella vulagris, Artemia salina y Daphnia pulex se pudo observar que la Chlorella vulgaris, si bien es la relevante desde los cuatro días, solo es necesaria hasta los 11 días, pues luego, la misma no fue considerada como alimento preferencial, sino el crustáceo branquiópodo del orden Anostraca, la Artemia salina, pero hasta los 25 días aproximadamente, ya que desde los 18 días, resultó equivalente con Daphnia pulex.

Asimismo, este crustáceo planctónico resultó la dieta preferencial a partir de los 32 días. Los cambios de la alimentación 
fueron ocasionados a partir de la tasa de crecimiento de los alevines de Odontesthes bonariensis donde la preferencia sobre la alimentación Artemia salina hasta los 25 días obedeció en lo fundamental al tamaño, ya que según Watanabe et al. (1983), la composición nutricional es muy similar.

Fue relevante observar el cambio de régimen alimentario de los peces. A mayor tamaño los peces requieren presas grandes; en este caso la Daphnia pulex fue apropiada a partir del día 25 , siendo tal comportamiento como depredación natural, coincidiendo con los estudios realizados por Sagretti y Bistoni (2001). Si bien, Odontesthes bonariensis posee un régimen alimenticio preferencial zooplanctófago con cambios ontogenéticos (Mancini et al. 2008) y estacionales (Grosman, 1995), adapta su dieta en base a la oferta realizando incursiones tróficas a diferentes comunidades alternativas como macrófitas (Ringuelet, 1940), (Sagretti y Bistoni, 2001). El pejerrey preda principalmente sobre los organismos vivos y la variación de la dieta es de acuerdo a la longitud alcanzada por la especie en mención, observándose evidente ictiófaga.

Penalillo \& Araya (1996), mencionan que el pejerrey en la etapa de larvas alimentados con plancton y nauplios de artemia tuvieron buenos resultados. Dicha mención se afirma con los resultados en la investigación. Mancini et al. (2004), Grosman 1995) refieren que el pejerrey en su régimen alimenticio es un pez planctófago, hasta $180 \mathrm{~mm}$, tal afirmación coincide con los resultados obtenidos en el presente estudio en donde el pejerrey resultó ser planctófago.

Wetzel y Likens (2000) sostienen que Odontesthes bonariensis basa su dieta en el zooplancton donde la depredación puede actuar como uno de los factores reguladores de la densidad de esta comunidad en ambientes lénticos. Pudo corroborarse en esta investigación que, a partir del día 11, la preferencia alimentaria sobre la Chlorella vulgaris fue nula, desplazándose a la Artemia salina por la Daphnia pulex después de los 25 días, interpretado por el posible mayor tamaño de esta última especie, ya que la depredación se hizo notable hasta aproximadamente los 46 días.

\section{Evaluación de crecimiento y supervivencia durante la etapa de larvas y alevines bajo condición de laboratorio}

$\mathrm{Al}$ analizar los ensayos con densidades poblacionales y condiciones de cultivo similares, mostraron resultados de longitud y peso con escasas variaciones durante el desarrollo corporal de los peces, exhibiendo crecimiento exponencial con relación al tiempo, alimentados de forma secuencial con (Chlorella vulagris, Artemia salina y Daphnia pulex). En la Figura 2, se observa el promedio de crecimiento de los tres ensayos $(\mathrm{cm})$ y el peso (g) de larvas y alevinos de pejerrey desde el primer día de nacimiento hasta los 46 días en donde se evidenció que el pez incrementó de talla mas no de peso. Este evento se relacionó con el consumo de alimento vivo. 


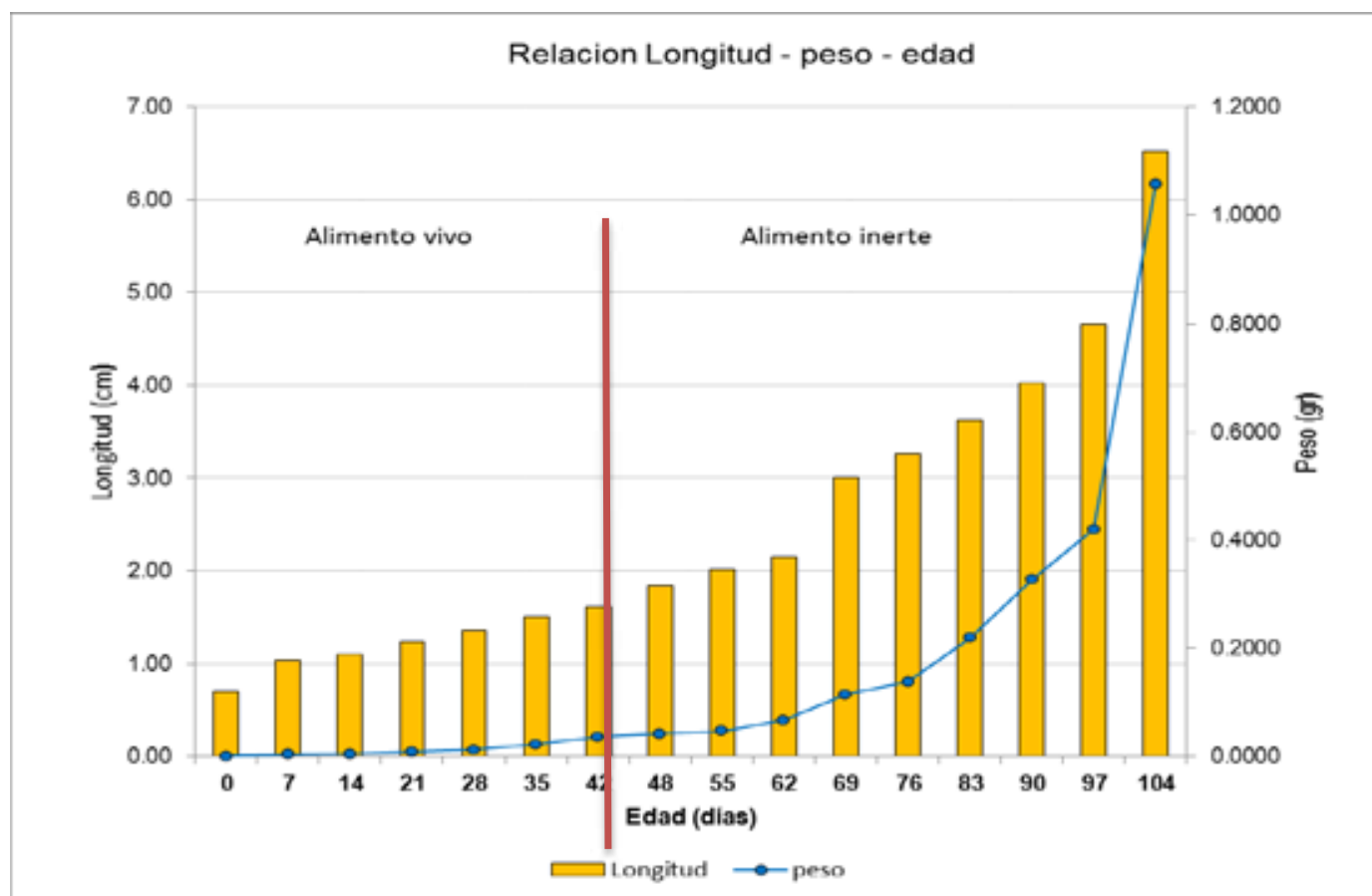

Figura 2. Promedio de crecimiento $(\mathrm{cm})$ e incremento de peso $(\mathrm{g})$ de alevinos de pejerrey después de recibir alimento Chlorella vulgaris, Artemia salina y Daphnia pulex en simultaneo, durante la primera semana de evaluación.

Así mismo, la Figura 3 muestra una ecuación exponencial alométrica por presentar un valor mayor a 3, con una correlación en donde el $\mathrm{R}^{2}$ es de 0.98 y la exponencial 3.046 el cual indica que los datos tabulados presentan una relación entre peso y talla del pejerrey mostrando un tipo de crecimiento alométrico positivo en los tres ensayos.

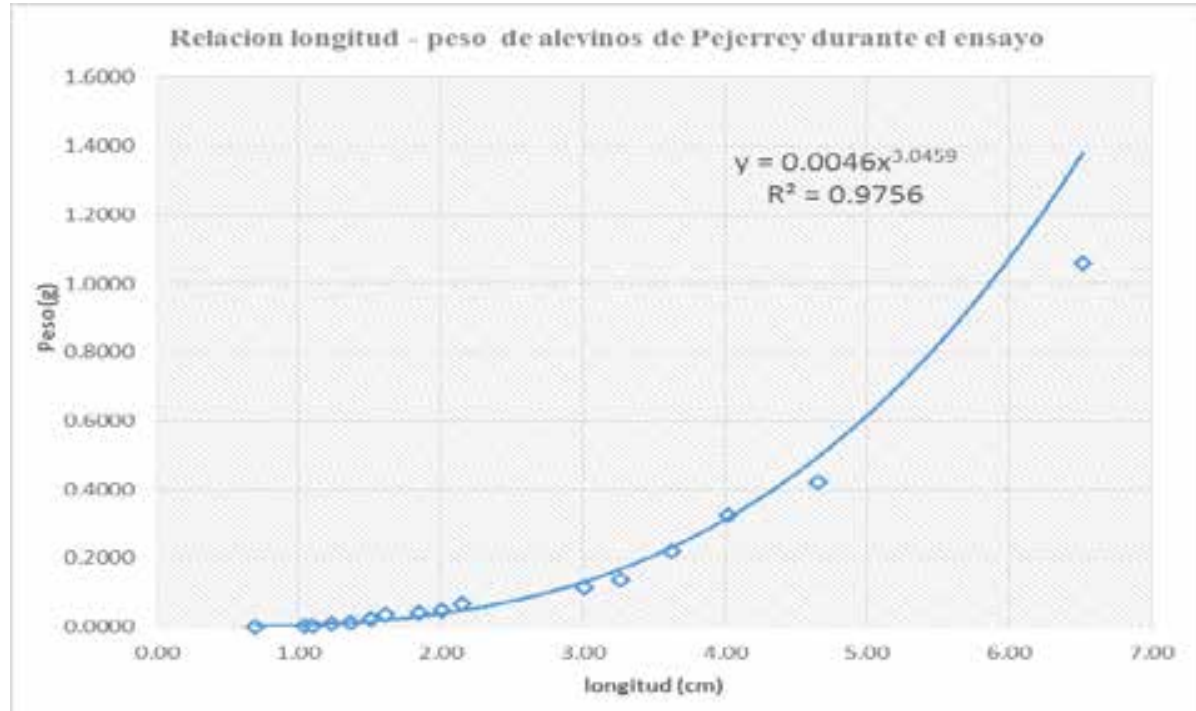

Figura 3. Análisis de ecuación exponencial durante el crecimiento de alevinos de pejerrey. 


\section{Índice de condición biológico}

Al analizar el índice de condición biológica de alevines de pejerrey (Odontesthesbonariensis) se halló quela talla y el peso no presentaron proporcionalidad hasta los 12 días; sin embargo, a partir del día 39, el índice de condición biológico fue proporcional. Después de este periodo se encontró, el IK tuvo un incremento significativo que pudo estar dado por el cambio paulatino de alimento vivo a alimento inerte. El índice de condición biológico del pez se mantuvo cercano a 1, (Tabla 9), quizás por cierta adaptación al suministro de alimento, pero a los 91 días, disminuyó y pudo deberse a un suministro limitado de alimento inerte. Así mismo, la comparación de medias de los días 52 y 65 fue la siguiente; para la media IK 52: $0.778 \pm 0.011$ con un intervalo de confianza al $95 \%$ y para IK 65: $0.535 \pm 0.005$ con un intervalo de confianza al 95.0\%.

Tabla 9

Indice de condijo biológico de pejerrey durante los 104 dias

\begin{tabular}{ccccccc}
\hline Día & Long $(\mathrm{cm})$ & DE & Peso $(\mathrm{g})$ & DE & IK & $\mathrm{N}^{\circ}$ \\
\hline 1 & 0.73 & 0.05 & 0.001 & 0.0002 & 0.26 & 24 \\
13 & 0.74 & 0.08 & 0.004 & 0.0004 & 0.89 & 24 \\
26 & 0.91 & 0.07 & 0.011 & 0.0016 & 1.43 & 24 \\
39 & 1.14 & 0.10 & 0.032 & 0.0061 & 1.43 & 24 \\
52 & 1.33 & 0.12 & 0.043 & 0.012 & 1.84 & 24 \\
65 & 1.94. & 0.31 & 0.095 & 0.041 & 1.29 & 24 \\
78 & 2.43 & 0.21 & 0.185 & 0.057 & 1.28 & 24 \\
91 & 3.20 & 0.42 & 0.389 & 0.132 & 1.19 & 24 \\
104 & 4.40 & 0.75 & 1.002 & 0.312 & 1.11 & 24 \\
\hline
\end{tabular}

Grosman et al. (2013), explican que los indicadores de condición corporal empleados en el pejerrey, poseen una pronunciada pendiente positiva hasta los $20 \mathrm{~cm}$ de longitud estándar, condición que se manifestó en el proceso de cambio de dieta de zooplanctófaga a ictiófaga. Se debe también señalar que el cambio de dieta sobre alimentación viva por inerte en este trabajo de investigación pudo influir en el incremento de peso del pez, ya que el cambio de alimento sucedió a los 46 días. En un estudio realizado por Velasco et al. (2014) señalan que, al finalizar el ensayo sobre el crecimiento de pejerrey, tuvo resultados en donde al inicio el IK fue de 1,09 y al final el IK fue 1,23 en 228 días, tal señalamiento guarda relación con el presente trabajo de investigación debido a que los resultados de IK se encontró cercanos a 1 , incluso en algunos casos superiores a 1 .

El crecimiento es una variable biológica que puede afectarse cuando las condiciones del medio y la alimentación resultan limitantes. En este caso, las condiciones del medio fueron favorables, ya que el manejo higiénico-sanitario se controló. Sin embargo, fue posible que existiera una ración alimentaria limitada en los últimos días, la cual pudo provocar periodos de ayunas en los ejemplares. La evolución en el coeficiente de condición pone de manifiesto la tendencia de los organismos a recuperar sus pesos corporales y cuando no 
se alcanza este factor de condición biológico, se indica el índice de adelgazamiento sufrido bajo cualquier situación estresante del medio; y, por ende, su capacidad depredadora. Si bien es cierto que la talla y el peso fueron incrementándose con cierta proporcionalidad el cual indica el buen estado del pez, incluso peces con IK mayores a 1. Así mismo, se observó que, a partir de los 78 días, se observó que el índice de condición disminuyó significativamente. Al parecer, el peso fue la variable que se vio más afectada y probablemente la acumulación de grasa corporal estuvo disminuida, aunque no se midió. El índice de condición resulta complejo compararlo con otras especies, ya que depende de las especies, estado de desarrollo, condiciones ambientales, intensidad y duración de los períodos de privación alimentaria, entre otros factores (egea et al. 2011).

Finalmente, pudo observarse que los individuos fueron adaptándose a la nueva dieta alimentaria. Tuvieron una leve disminución del índice de condición en los 23 últimos días, que fue atribuida al poco suministro de gramos de alimento por individuos.

\section{Estimación de tasa de crecimiento especifico (TCE) en porcentaje día}

Se tomaron muestras de alevines de pejerrey en los tres ensayos cada trece días. Durante 104 días se evaluó la longitud total (LT) en $\mathrm{cm}$ con un Ictiómetro, mientras el peso se controló con una balanza analítica en gramos (g). Con estos datos, se estimó la tasa de crecimiento específico TCE (\% día) que expresa el crecimiento del pez en peso diario influenciado por el espacio, alimento y temperatura. Se obtuvo valores de TCE de $9.90 \%$ en los primeros 13 días, el valor encontrado fue considerado el más alto en este ensayo, a los 26 días la TCE fue de $4.15 \%$, a los 39 días la TCE se encontró en $2.71 \%$; sin embargo, a los 52 días la TCE presentó el valor más bajo $(0.60 \%)$. Posiblemente haya sido influenciado por el cambio de alimento (alimento vivo a inerte). A partir del día 65 a 104 días el valor de TCE presentó un incremento manteniéndose cercanos a 1. Probablemente, el pez se habituó con el alimento inerte (Tabla 10).

Tabla 10

Tasa de crecimiento de pejerrey (TEC) durante 104 dias

\begin{tabular}{ccccccc}
\hline Día & Long $(\mathrm{cm})$ & DE & Peso $(\mathrm{g})$ & DE & TCE $\%$ & $\mathrm{~N}^{\circ}$ \\
\hline 1 & 0.73 & 0.05 & 0.001 & 0.0002 & & 24 \\
13 & 0.74 & 0.08 & 0.004 & 0.0004 & 9.90 & 24 \\
26 & 0.91 & 0.07 & 0.011 & 0.0016 & 4.15 & 24 \\
39 & 1.14 & 0.10 & 0.032 & 0.0061 & 2.71 & 24 \\
52 & 1.33 & 0.12 & 0.043 & 0.012 & 0.60 & 24 \\
65 & 1.94 & 0.31 & 0.095 & 0.041 & 1.30 & 24 \\
78 & 2.43 & 0.21 & 0.185 & 0.057 & 0.85 & 24 \\
91 & 3.20 & 0.42 & 0.389 & 0.132 & 0.68 & 24 \\
104 & 4.40 & 0.75 & 1.002 & 0.312 & 0.97 & 24 \\
\hline
\end{tabular}


Estos resultados se asemejan a los obtenidos por Velasco et al. (2014), quienes registraron TCE entre 2.1\%, $0.5 \%$ y $1.3 \%$ de crecimiento diario. Para el estudio realizaron ensayos con larvas de Odontesthes bonariensis provenientes de desoves naturales, posteriormente fueron criados en tanques circulares, mientras Orellana \& Toledo (2006), reportan en su investigación realizada en sistema de jaulas flotantes en el mar con Odontesthes regia, que la TCE obtenida para todo el periodo fue de $0.6 \%$ día. Los resultados obtenidos por los autores en mención son inferiores a los obtenidos en el presente trabajo de investigación, probablemente, por el tipo de alimento y los factores ambientales como; (temperatura, oxígeno y pH), así mismo Grossman et al (2013) menciona en su estudio sobre condición, alimentación y crecimiento del pejerrey Odontesthes bonariensis que el cambio de dieta zooplanctófaga a piscívora, estaría también influyendo en la modalidad de crecimiento. Solimano et al. (2014) realizó una comparación entre el cultivo intensivo y extensivo de pejerrey en donde menciona que, en el caso de los cultivos en jaulas flotantes, se observa un fuerte incremento de la TCE, posiblemente sea por la intervención de la mano del hombre en manejo y suministro de alimento balanceado, así mismo la temperatura influye en el desarrollo del pez que puede generar crecimiento o retardo.

\section{Conclusiones}

Al suministrar Chlorella vulgaris como alimento a larvas y alevinos de pejerrey Odontesthes bonariensis, la viabilidad larvaria fue mayor hasta el día 11, pero relevante los primeros siete días, considerando como fuente directa de alimento, por presentar micronutrientes y suplemento de enzimas exógenas. La Artemia salina se consideró como segundo alimento vivo con $70 \%$ de viabilidad larvaria, mientras la Daphnia pulex en los primeros días no fue considerada como alimento preferencial; sin embargo, a partir del día 11 se evidenció como alimento principal.

En la frecuencia preferencial alimentaria según el análisis del sistema digestivo se encontró Chlorella vulgaris en mayor porcentaje los primeros cuatro días, pero, se considera necesario hasta el día 11. La Artemia salina fue considerada como alimento preferente desde el 12 hasta el día 18 y la Daphnia pulex resultó ser alimento preferencial a partir del 32 hasta el 46 día.

Los alevinos de Odontesthes bonariensis demandan diferencias en el tipo y la calidad de alimento según la edad. Así mismo, el crecimiento alcanzado hasta la etapa de alevines bajo el orden de dieta suministrado y bajo el control de factores físico- químicos del agua mostró que el índice de condición (IK), en los primeros días fue mayor a 1. Posteriormente se comportó con variaciones que se pudieron deber a un cambio paulatino de alimento. La tasa de crecimiento específico (TCE) en general se mantuvo cercana a 1 , lo cual indica que los valores de talla y peso se encontraron en condiciones normales. Así mismo, la supervivencia en el ensayo 1 fue $96.1 \%$, durante el ensayo 2 fue $96 \%$ y en el ensayo $3,97.5 \%$. 


\section{Referencias}

Bahamondes, I., Soto, D., \& Vila, I. (1979). Hábitos alimentarios de los pejerreyes (Pisces: Atherinidae) del embalse Rapel, Chile.; [Food habits of three Atherinidae fishes from Rapel pound. Chile [Basilichthys australis, Ondonthesthes bonariensis Valenciennes, Odontesthes mauleanum]]. Medio Ambiente (Chile)..(4(1), 3-18.

Bdui, S. (1988). Diccionario de tecnología de los alimentos. Editorial Alhambra Mexicana, México, D.F. 300.

Burbidge, R. G., Carrasco, M. C., \& Brown, P. A. (1974). Age, growth, length-weight relationship, sex ratio and food habits of the Argentine pejerrey, Basilichthys bonariensis (Cuv. and Val.), from Lake Peńuelas, Valparaíso, Chile. Journal of Fish

Cuvier y Valenciennnes (1835). Historie Naturelle des Poisssons X, 1-358.

Chura-Cruz, R., Cubillos, S., Luís, A., Tam, M., Segura, Z., \& Villanueva, Q. (2013). Relación entre el nivel del lago y la precipitación sobre los desembarques del pejerrey Odontesthes bonariensis (Valenciennes, 1835) en el sector peruano del Lago Titicaca entre 1981 Y 2010. Ecología aplicada, 12(1), 19-28.

Dabrowski, K., (1984). The feeding of fish larvae: present «state of the art» and perspectives. Reproduction Nutrition Développement, 24(6), 807-833..
Del Ponti, O. \& Garcia, M. (2015). Alimentación del pejerrey luego de una mortandad masiva de Odontesthes bonariensis en las lagunas Bajo Giuliani y Don Tomás - Provincia de La Pampa, Argentina. Semiárida Rev. Fac. Agron. UNL Pam Vol. 25(1), 7-15.

Egea Nicolás, M., Rueda González, F., Martínez López, F., \& García García, B. (2011). Efecto de la realimentación tras un periodo de ayuno sobre el crecimiento en el sargo picudo Diplodus puntazzo (Cetti, 1777). Boletin. Instituto Español de Oceanografía, 18(1-4), 357-362.

Govoni, J.J., Boehlert, G.W. \& Watanabe, Y. (1986). The physiology of digestion in fish larvae. Environmental Biology of Fishes, 16(1-3), 59-77.

Grosman, F. \& Sergueña, S. (1996). Parámetros biológicos y sociales de una pesquería deportiva de pejerrey.Odontesthes bonariensis En: Actas VI Jornadas Pampeanas deficiencias naturales, 133-141.

Grosman, F., Sanzano, P., Colasurdo, V. (2013). Condición, alimentación y crecimiento del pejerrey Odontesthes bonariensis en una laguna pampeana de Argentina. Revista AquaTIC; 39, 44-54.

Grosman, F., Sanzano, P., González, G., Agüería, D. \& Sergueña, S. (2001). Ecología reproductiva, 
edad, crecimiento, condición y alimentación del pejerrey Odontesthes bonariensis en un ambiente del SO de la provincia de Buenos Aires, Argentina. Aqua TIC, 12, 1-24.

Grosman, M.F. (1995). Variación estacional en la dieta del pejerrey Odontesthes bonariensis .Rev. Asoc. Cs. Nat. Litoral, 26, 9-18.

Grosman, M.F. (2005). Variación Estacional en la Dieta de Pejerrey Odontesthes Bonariensis. Natura Neotropicalis, 1(26), 9-18.

Instituto del Mar del Perú (2017). Anuario científico tecnológico IMARPE 2017. Vol 17. Correo electrónico: imarpe@imarpe.gob. pe;biblioteca@imarpe.gob.pe

López, H. L., García, M. L., \& Grosman, F. (2001). Aspectos históricos e importancia regional del pejerrey bonaerense.

Orellana, F. A., \& Toledo, H. E. (2007). crecimiento de juveniles de pejerrey (Odontesthes regia Humboldt, 1821)(Atherinidae) en balsas jaulas. Gayana (Concepción), 71(1), 76-83.

Reeve, M.R. (1963). The filter- feeding of Artemia. II. In suspension of various particles. J. exp. Biol., 40(1): 207-214.

Ricker, W. (1979). Growth rates and models. En: W. Hoar, D. Randall, J. Brett, Edss. Fish Physiology. Volume VIII, Bioenergetics and
Growth. Academic Press, New York, USA, 677-743.

Ringuelet, R. A. (1940). Ecología alimenticiadel pejerrey (Odonthestes bonariensis). Revista del Museo de la Plata, 2(17), 427-461.

Ringuelet, R. A., Iriart, N. R., \&Escalante, A. H. (1980). Alimentación del pejerrey (Basilichthys bonariensis bonariensis, Atherinidae) en laguna Chascomús (Buenos Aires, Argentina). Limnobios, 1.

Sagretti, L. and M. d. 1. A. Bistoni (2001). Alimentación de Odontesthes bonariensis (Cuvier y Valenciennes 1835) (Atheriniformes, Atherinidae) en la laguna salada de Mar Chiquita (Córdoba, Argentina)." Gayana (Concepción) 65(1), 37-42.

Solimano, P. J., García de Souza, J. R., Maiztegui, T., Bazzani, J. L., Baigún, C. R. M., \& Colautti, D. C. (2014). Evaluación de modelos de crecimiento de diferentes condiciones de cultivo de pejerrey (Odontotesthes bonariensis). Biología Acuática.

Statgraphics Plus For windows: SGPW. (2001). Version 5.1. Copyright 1994-2001 for Statistical Graphics Corporation.

Tucker, J. W. Jr. (1998) Marine fish culture Kluger Academic Publishers, Massachusetts, E.U.A. 750.

Velasco, C., Berasain, G. \& Padín, D. (2014). Crecimiento de juveniles 
de pejerrey Odontesthes bonariensis bajo dos diferentes condiciones de cultivo. Biología Acuática, 30.

Vila, I., \& Soto, D. (1984). Odontesthes bonariensis "pejerrey argentino", una especie para cultivo extensivo. FAO Documento técnico, 4, 224-228.

Wetzel, R., \& Likens, G. (2000). Limnological analyses, 3 (ed) Springer Verlag. Nueva York.
Wetzel, R.G. (2001). Limnology. Lake And River ecositems. Thrid Edition. Academic Press. U.S.A. 1006.

Yujra Flores, E., Amaru Chambilla, G., Segura Zamudio, M., Villanueva Quispe, C., Siguayro Mamani, H. y Chura Cruz, R. (2016). Obtención experimental de alevines de Odontesthes bonariensis (pejerrey), bajo manejo productivo en cautiverio y laboratorio, PunoPerú. Campus, (21),49-56. 
